\title{
Large scale high strain-rate tests of concrete
}

\author{
M. Peroni, G. Solomos, B. Viaccoz, G. Magonette, and R. Kiefer \\ European Commission, Joint Research Centre, IPSC/ELSA, 21027 Ispra, Italy
}

\begin{abstract}
This work presents the stages of development of some innovative equipment, based on Hopkinson bar techniques, for performing large scale dynamic tests of concrete specimens. The activity is centered at the recently upgraded HOPLAB facility, which is basically a split Hopkinson bar with a total length of approximately $200 \mathrm{~m}$ and with bar diameters of $72 \mathrm{~mm}$. Through pre-tensioning and suddenly releasing a steel cable, force pulses of up to $2 \mathrm{MN}, 250 \mu$ s rise time and $40 \mathrm{~ms}$ duration can be generated and applied to the specimen tested. The dynamic compression loading has first been treated and several modifications in the basic configuration have been introduced. Twin incident and transmitter bars have been installed with strong steel plates at their ends where large specimens can be accommodated. A series of calibration and qualification tests has been conducted and the first real tests on concrete cylindrical specimens of $20 \mathrm{~cm}$ diameter and up to $40 \mathrm{~cm}$ length have commenced. Preliminary results from the analysis of the recorded signals indicate proper Hopkinson bar testing conditions and reliable functioning of the facility.
\end{abstract}

\section{Introduction}

The design and assessment of concrete structures subjected to impact loads requires the knowledge of the constituent materials behaviour at higher strain-rates. Concerning concrete, despite the effort and experimental work carried out so far, the data available are still relatively few. The reason of the lack of experimental data could originate from the complexity of the relevant experiments, in addition to the fact that these tests are not always directly comparable to each other, even for the simpler compression and tension tests. Often the data have been obtained by different experimental techniques and in dynamic testing such differences can be more marked because of the wave propagation and inertia effects [1]. For this reason it is essential that apparatuses capable of handling the wave propagation be used for the mechanical characterization of concrete at high strain-rates. It is the intention of this work to analyse the development and present some results of an innovative equipment, based on Hopkinson bar techniques, for performing dynamic compression tests on concrete specimens of large size. The activity has been centered at the HOPLAB facility (of the Joint Research Centre at Ispra, Italy), which is basically a large-scale split Hopkinson bar. This facility is used for the study of materials and structural components to fast dynamic loads, such as those due to blasts and impacts. The problem to be eventually investigated concerns more specifically the dynamic characterization of concrete typical of dams, where very large size aggregates $(>100 \mathrm{~mm}$ ) may be found. Thus large specimens of representative volume have to be tested. To this end concrete specimens of maximum aggregate size of up to $60 \mathrm{~mm}$ have been produced, which in turn have required a minimum dimension of the specimens of approximately $200 \mathrm{~mm}$. To investigate the compression properties and to check the influence of the hydrostatic tensor, dynamic compression of cylindrical and confined cylindrical specimens has been planned to be conducted with specimens of $200 \mathrm{~mm}$ diameter and of up to $400 \mathrm{~mm}$ length. These are huge specimens for Hopkinson bar testing, and a particular setup of two twin bars has been developed in order to apply dynamic compression to the specimen starting from a tensile propagating wave.

\section{Experimental facility}

The HOPLAB facility is probably one of the biggest existing Hopkinson bar apparatuses with a length of more than $200 \mathrm{~m}$ and of bar diameters of $72 \mathrm{~mm}$ made of high-strength steel. Through pre-tensioning and suddenly releasing a steel cable, rectangular force pulses of up to $2 \mathrm{MN}, 250 \mu \mathrm{s}$ rise time and $40 \mathrm{~ms}$ duration can be generated and applied to the specimen tested [2]. The cable pre-tensioning is effected with a hydraulic jack, electronically controlled, and the instantaneous release of its opposite end is achieved through breaking of a fragile bolt, which resists all the pretension. The fracture of the bolt is induced by a small detonation of explosive inserted in it. This high-strength cable is $100 \mathrm{~m}$ long and has an equivalent diameter of $72 \mathrm{~mm}$. The tensile pulse generated is transmitted to the actual input bar, to the specimen and to the output bar (about $90 \mathrm{~m}$ long). At the distal end of the output bar a hydraulic damper allows to dissipate the remaining energy emanating from the cable and transferred through the specimen to the output bar.

Clearly, in its "natural" configuration the HOPLAB can deliver dynamic tensile pulses in a straight-forward manner, figure 1 . By changing the central part of the equipment it is possible to exploit the tensile wave generated, and to perform different kinds of tests, as for example compression tests. With reference to this last consideration, the next sections describe the main features of an experimental setup developed to carry out dynamic compression tests on large scale specimens of concrete.

\section{Dynamic compression configuration}

\subsection{Preliminary numerical study}

As shown in figure 2, the basic element of the new configuration is the introduction of the twin incident and 


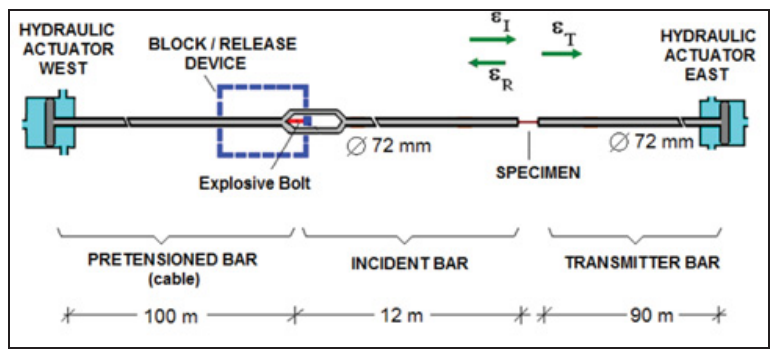

Fig. 1. Sketch of the HOPLAB facility.

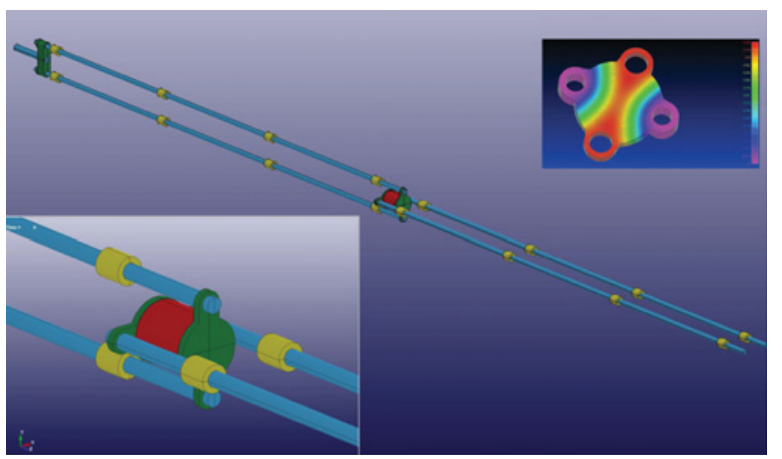

Fig. 2. Numerical model of equipment setup with details of the specimen zone and of the compression plates.

transmitter bars with the strong steel plates at their ends, where large specimens can be mounted and compressed. With this "motion inversion frame" the available tensile pulse is transformed into a compressive one on the specimen, which is thus crushed dynamically.

However, there is a considerable departure from the standard one-dimensional geometry, which is inherent in the analysis of the Hopkinson bar technique [3,4]. This is due to both the distance between the twin bars $(270 \mathrm{~mm})$ and to the flexibility of the end plates. For this reason a numerical study of the new configuration has accompanied all steps of its development. Both explicit and implicit finite element analyses have been performed and their results have led to optimised geometrical shapes and distribution of the extra masses. An example is shown in figure 2. These simulations have indicated that a Hopkinson bar type test would be reliably reproduced.

\subsection{Mechanical structure}

As mentioned above, the developed setup should be able to perform high strain-rate compression tests on large size specimens of concrete with a diameter of $200 \mathrm{~mm}$ and a maximum length of $400 \mathrm{~mm}$. As shown in figure 3, this objective has been materialised by a setup based on a "dynamic inversion frame" that transforms the tensile pulse into a compression load applied to the specimen. The inversion frame is composed of two couples of twin bars, which are placed at the four vertices of a square and move in opposite directions. These bars are made of high-strength stainless steel and are $6 \mathrm{~m}$ long and of $60 \mathrm{~mm}$ diameter.

The compressive load is applied to the specimen by means of two plates (also made of high-strength steel),

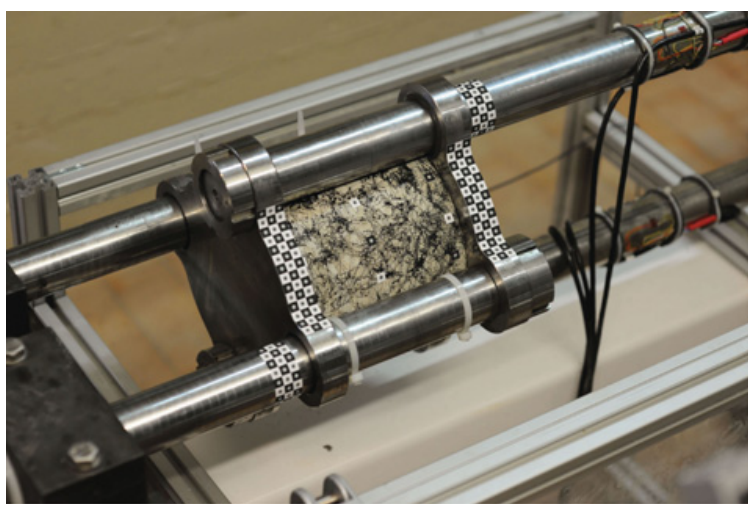

Fig. 3. Inversion frame setup - specimen clamping.

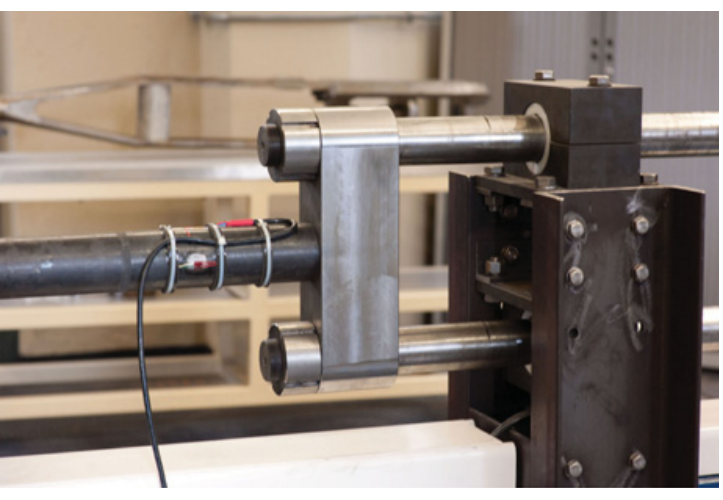

Fig. 4. Inversion frame setup - input fork.

which also "keep together" the four bars. Input and output twin bars are rigidly connected to their own plate and can slide in the two plastic bearings of the other plate. This solution allows to obtain a very stiff mechanical structure and to minimise oscillations during the test. To reduce friction all bars slide on Teflon bearings that are supported on high-stiffness metallic structures, as shown in figure 4. The same figure also shows the input fork that connects the single input bar ( $72 \mathrm{~mm}$ diameter) to the input twin bars. As discussed above, the design of this "branching" has been investigated with numerical simulations in order to avoid spurious reflections and to limit the bending of the twin bars. The connection between the output twin bars and the single output bar ( $72 \mathrm{~mm}$ diameter) has been made with mechanical components similar to those of the input fork, in a way that does not constrain the axial displacement of the single output bar, figure 5. It is thus possible to place an energy absorber between the twin and the single bars in order to dissipate part of the energy transmitted by the specimen to the output bars.

Figure 5 shows the energy absorber (an aluminium tube) before and after the test. A large amount of energy has been absorbed by the plastic deformation of the aluminium tube (through fold formation and axial collapse), and it is to be noted that this device has never failed to work efficiently.

\subsection{Actuators and dampers}

As explained above, the input pulse is generated by pretensioning the input cable with a hydraulic jack, which 

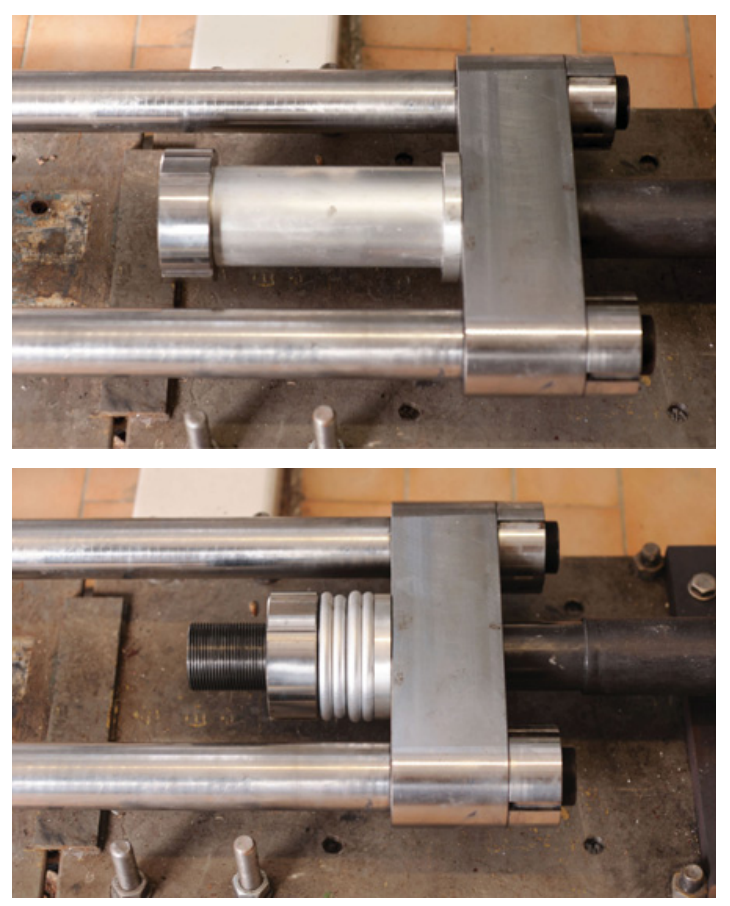

Fig. 5. Inversion frame setup - mechanical damper before and after the test.

is electronically controlled. The management of this liberated elastic energy is a crucial issue, as its value can be in excess of $1 \mathrm{MJ}$. Part of it is consumed at the specimen, another part at the previously described aluminium tube absorber, but a considerable fraction of it may still end up at the actuators. Thus the equipment needs energy damping mechanisms at the end of the input and output bars to absorb part of the energy initially stored in the cable, and to also stop the movement of the bars. The problem is not as acute for the pre-tensioned cable because it is selfrelaxing after its release. These roles have been assigned to two hydraulic jacks that can work at the same time both as actuators and dampers. The two cylinders have been developed entirely at the ELSA lab of the JRC and their main features include: piston of $400 \mathrm{~mm}$ diameter, $1 \mathrm{~m}$ stroke, tubular shaft (external diameter $160 \mathrm{~mm}$, internal diameter $80 \mathrm{~mm}$ ), maximum static working pressure 320 bars. All the mechanical components of the cylinder have been made of high-strength steel and the jack is electronically controlled with a proportional valve that connects its two chambers. Five additional bypasses allow the increase of the oil flux between the two chambers when the cylinder works as a damper.

The two actuators/dampers differ only in a few features. The "West" cylinder, figures 1 and 6, usually works as actuator for stretching the input cable. The connection between the input cable and the cylinder is rigid and six gas accumulators allow the compensation of the pressure lack of balance when the cable is abruptly unloaded. The "East" cylinder works as actuator only during the test preparation phase, when there is need to move the output bar in order to properly position the specimen. When a dynamic test takes place all its bypasses are opened in order to use this cylinder as a damper and to absorb the energy transmitted there by the output bar. To reduce the pressure peaks, six

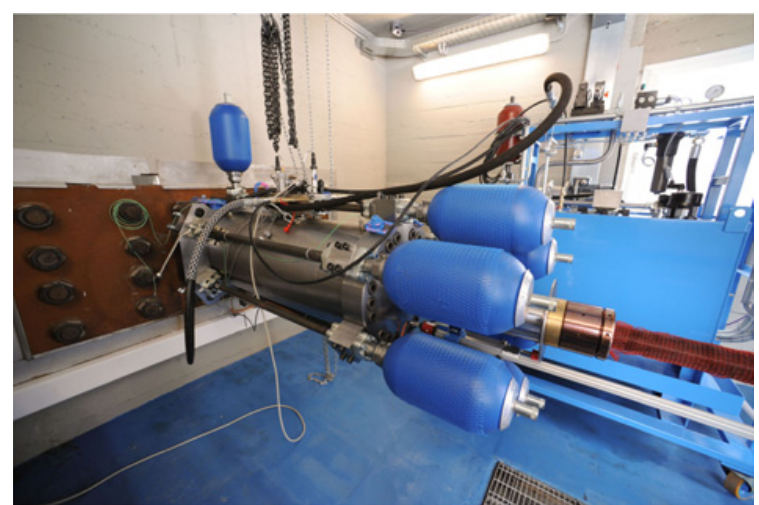

Fig. 6. West cylinder and hydraulic pumping station.

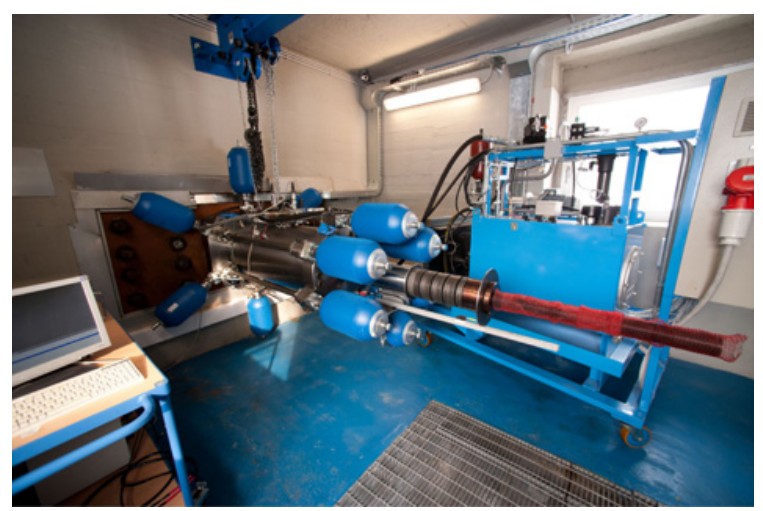

Fig. 7. East cylinder and hydraulic pumping station.

gas accumulators have been installed on each chamber, figure 7. The connection between the output bar and the jack is elastic (a series of Belleville springs have been mounted). The two independent hydraulic power units have been manufactured by Moog, while the control of the two jacks is performed with an electronic controller developed in ELSA. This allows to impose the pressurisation and displacements in the actuators remotely from the control room.

\subsection{Instrumentation}

The HOPLAB facility requires a high sampling-rate transient recorder capable of acquiring electrical signals in three different areas: west end, east end and the specimen zone. This requirement has been fulfilled by employing three different transient recorders (Nicolet Multipro with a maximum sampling-rate of $1 \mathrm{MHz}$ ) synchronized with the electrical signal that triggers the explosion of the fragile bolt. In addition, in order to effectively study the dynamic behaviour of the different parts of the equipment (especially in the development phase) and of the tested specimens, a high-speed camera (synchronized with the transient recorders) has been intensively used. This one is an IDT-Y4 high-speed camera able to perform $4000 \mathrm{fps}$ at maximum resolution. The acquisition frame-rate can be increased to $200000 \mathrm{fps}$ by reducing substantially, however, 


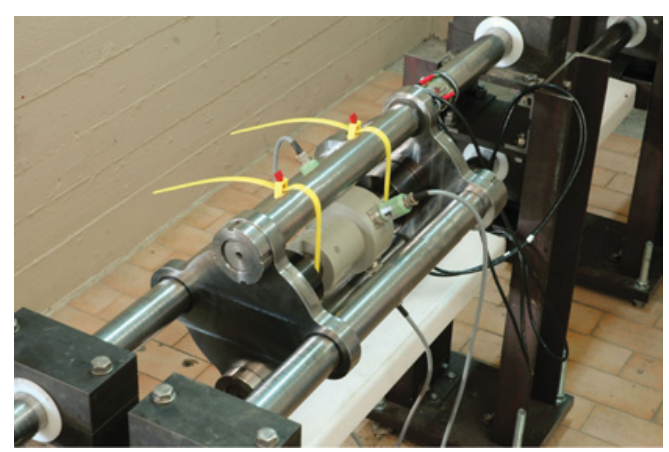

Fig. 8. Static calibration with an HBM reference load cell.

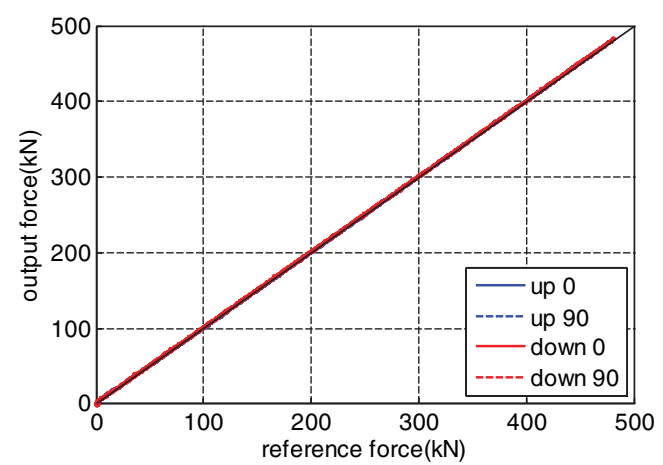

Fig. 9. Static calibration of strain-transducers (section 4).

one dimension of the photo acquired. As will be discussed in the next paragraph, high speed photo sequences allow to obtain full-field displacement measurements by using Digital Image Correlation techniques (DIC).

Further, both east and west actuators have been instrumented with static (WIKA) and dynamic (Kistler) pressure transducers, and with static (MTS temposonics) and dynamic (SpaceAge Control wire potentiometers) displacement transducers. In the specimen zone the bar strain histories have been recorded in several positions with strain-gage sensors, in which a full-bridge setup has been adopted to compensate for bending and thermal phenomena. The captured signals are conditioned with a high-speed strain-gage conditioner (Vishay 2400 with cutoff frequency of $100 \mathrm{kHz}$ ). The twin input bars have been each instrumented with three measurement points along their length and the output bars with two. At each such point two transducers have been placed, at orthogonal diameters, in order to eliminate bending phenomena and to have measurement redundancy. In addition piezoelectric force transducers (Piezotech PVDF Shock Gauges) have been placed between the specimen and the loading plates in order to measure the local pressure developed at these interfaces.

\section{Calibration and preliminary tests}

To check the correct alignment of the equipment and to calibrate the strain-gages mounted on the bars a series of tests have been performed. In practice, by using the two

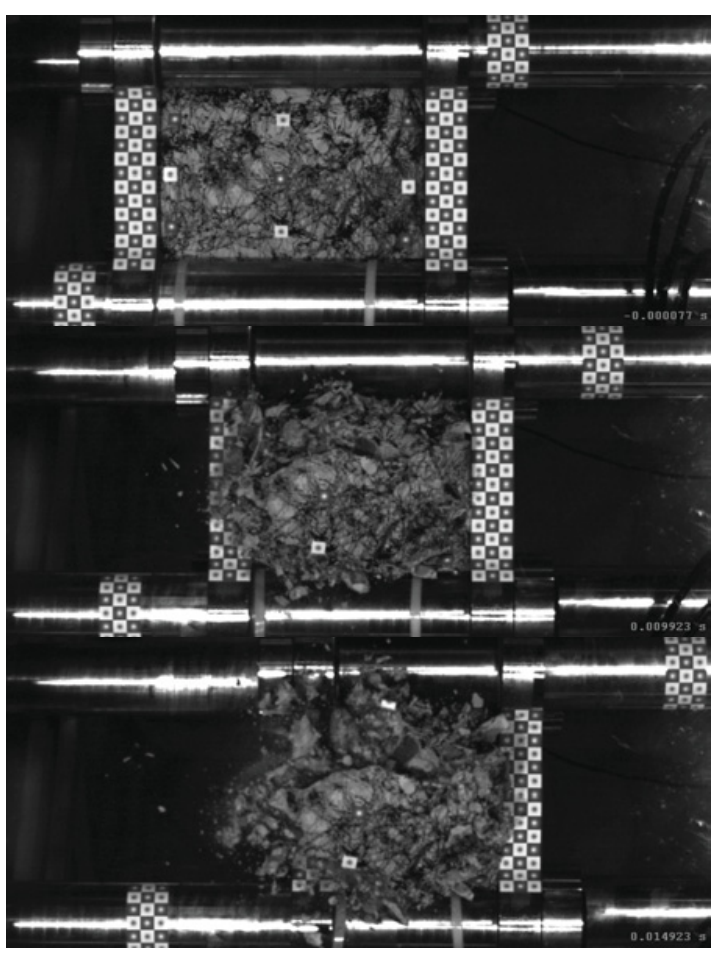

Fig. 10. High speed photo sequence of compression test.

actuators at the east and west ends, the full equipment has been put in tension with a reference load cell placed between the two loading plates, as shown in figure 8 .

Comparing the output signals of the reference load cell and the bar strain recordings an accurate calibration has been performed and no bending phenomena have been encountered with loads in excess of $1 \mathrm{MN}$.

Figure 9 shows an example of the comparison between the four signals acquired at the last measurement location of the twin input bars (section 4). The four signals are correctly overlapped and non-linear trends due to bending phenomena are completely absent. At this stage a series of experiments have been performed to evaluate the performance of the developed equipment with actual cylindrical concrete specimens. As mentioned, their dimensions are: $200 \mathrm{~mm}$ diameter and 200 and $400 \mathrm{~mm}$ length. The maximum pre-tensioning reached in this series of experiments is about $2 \mathrm{MN}$, which in turn generates an input pulse of $1 \mathrm{MN}$ amplitude. The velocity of the loading plates with these loads is in the range of $10 \div 15 \mathrm{~m} / \mathrm{s}$. Figure 10 shows a photo sequence acquired with the highspeed camera at $15000 \mathrm{fps}$ from the compression test of a concrete specimen of $200 \mathrm{~mm}$ diameter and $200 \mathrm{~mm}$ length. The black/white markers on the equipment and the specimen allow to determine with sufficient precision the displacements during the test using DIC techniques, while the irregular patterns on the specimen will be used for determining the specimen strain field. The concrete specimens fail in about $1 \mathrm{~ms}$ and failure is not always localised near the input loading plate (the left one in figure 10). This observation may serve as a guarantee that the specimen reaches the force equilibrium during the test, as will be shown in more detail below. 


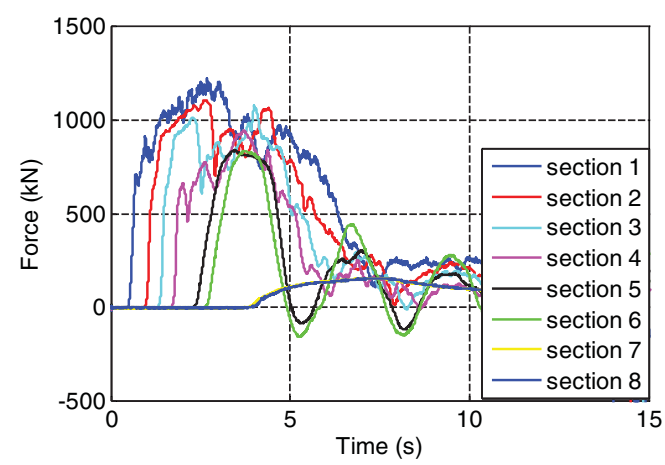

Fig. 11. Example of signals recorded during a compression test.

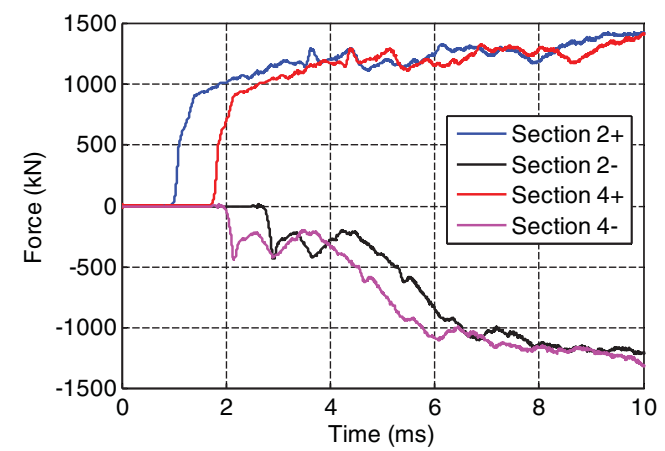

Fig. 12. Separated waves on input twin bars.

\section{Result analysis}

The standard equations, which involve incident $\varepsilon_{\mathrm{I}}$, reflected $\varepsilon_{\mathrm{R}}$ and transmitted $\varepsilon_{\mathrm{T}}$ strain waves, are used. As in the classical Hopkinson tests, the elaboration of the bar strain histories to arrive at the stress-strain diagram of the specimen is not trivial, and in order to obtain reliable results some correction algorithms must be applied. In addition, in this case the non standard geometry (the machine is not properly uni-axial) and the large dimensions of structure and specimens tend to compound potential complexities in data processing.

An idea of the bar strain histories is provided in figure 11, which presents the trends of the forces as recorded (proportional to the strains acquired) at different sections along the bars of the apparatus. The signal acquisition is synchronized with the explosion of the fragile bolt. During the test eight measurement points have been acquired: section 1 on the single input bar before the input fork, sections 2, 3 and 4 on the twin input bars, sections 5 and 6 on the twin output bars and sections 7 and 8 (these signals in practice coincide) on the single output bar after the mechanical energy absorber.

For what concerns signals of the twin bars, the force plotted is the sum of the forces of the two bars at the corresponding strain-gage stations. As it is immediate to observe, the incident and reflected waves that travel in the input bars overlap, since the strain pulse $(40 \mathrm{~ms})$ is definitely longer than the traversing time of the input twin bars. This phenomenon can also occur in the output twin bars if the specimen does not collapse in the first instants of the test. Signals recorded on the single output bar represent

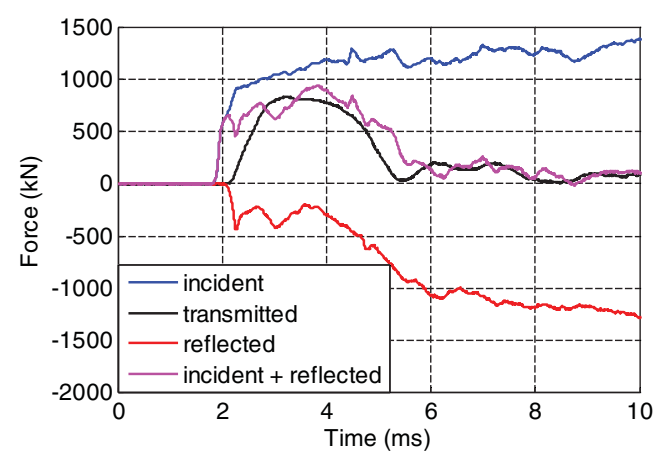

Fig. 13. Equilibrium check.

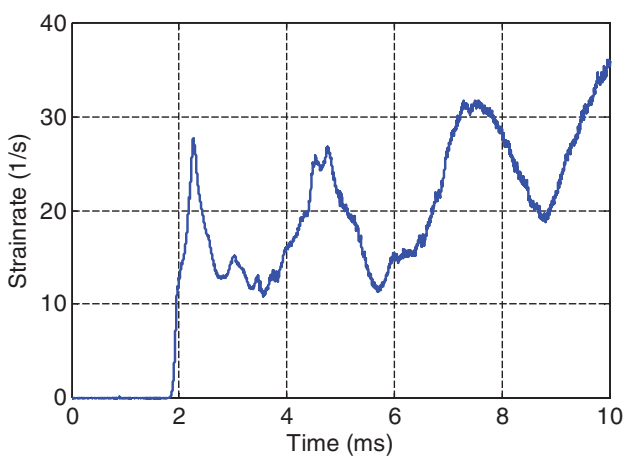

Fig. 14. Strain-rate trend in a compression test.

the load transmitted to the output equipment end at the east actuator/damper.

The first step of data processing is to separate waves that travel in opposite directions in the bar and to check the equilibrium at the two specimen ends. Doing this, the procedure proposed by [5] has also been used to compensate eventual distortions due to dispersion phenomena (justified here due the large cross-section of the equipment bars). Figure 12 shows the separated waves in the input twin bars (signs + and - represent ascending and descending waves). Applying next a time shifting and compensating for distortion due to dispersion [6], it is possible to make the equilibrium check at the specimen ends, as shown in figure 13. It is seen that, although the specimen is quite long, the two forces corresponding to the "transmitted wave" and "incident + reflected waves" have essentially the same trends and magnitude. Figure 14 shows the curve of strain-rate during the test. As expected the strain rate is not perfectly constant due to the brittle behaviour of this kind of material. The variations, however, are quite small to substantially influence the material behaviour.

Figure 15 finally presents the stress-strain curve of the specimen tested. To obtain this last curve the assumption of uniform stress and strain fields in the specimen has been made. In addition, the static deflection of the loading plates has been compensated (using the data obtained from the numerical simulations described above) according to the principles presented in [7].

As it may be expected, even with this kind of corrections the slope of the rising part of the stress-strain curve is rather small when compared with the static data. This is mainly due to the fact that this apparatus, and in general Split Hopkinson Pressure Bar testing, does not produce 


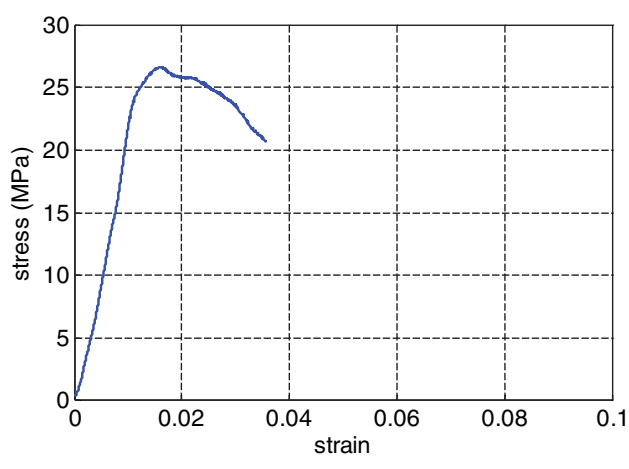

Fig. 15. Stress-strain curve of concrete specimen.

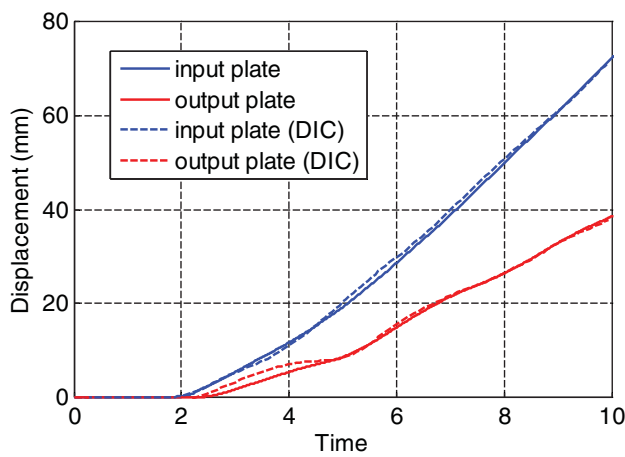

Fig. 16. Comparison of displacements applied to loading plates.

very accurate results in the small strain regime. This aspect is exalted in the developed setup due to the presence of the twin bars and the low stiffness of the loading plates (compared with the axial stiffness of the bars). To verify the accuracy of the current data-processing the last two figures show the comparison between data obtained with the Hopkinson elaboration techniques and data acquired with additional transducers placed on the specimen. Figure 16 presents the comparison of displacements obtained with the data processing described before and with DIC algorithms applied to the high speed photo sequence of the test. As observed, the two curves differ substantially in the first instants of the test (dominated by elastic strains), while the accuracy of Hopkinson data elaboration improves later when the level of strain increases. Figure 17 on the other hand shows the comparison between the force

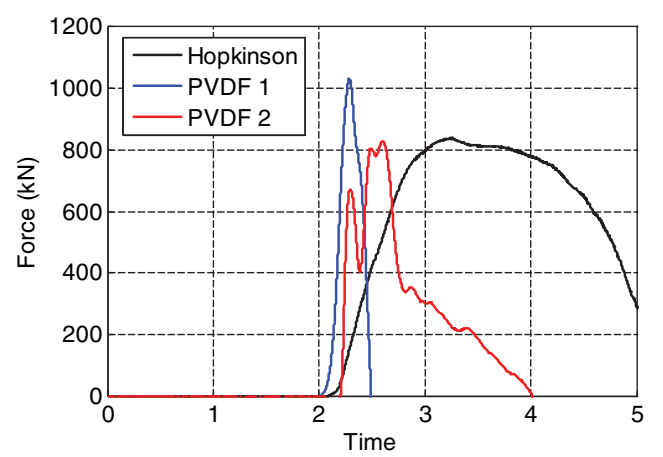

Fig. 17. Comparison of forces (output side of the specimen).

applied at the output face of the specimen, as measured with two piezoelectric PVDF sensors, and the trend obtained with the above Hopkinson analysis techniques.

The signals obtained from the PVDF sensors are very punctual (the active surface of the sensors is $5 \times 5 \mathrm{~mm}$ ) and their measures are strongly influenced by the presence of aggregates in the concrete specimen just ahead of them. Clearly the signal obtained with standard Hopkinson data processing is smoother, due to the presence of the additional masses of the loading plates. Despite these considerations, the maximum levels of the load applied appear to be perfectly compatible.

The above first results confirm that this new apparatus is overall capable of delivering dynamic compression testing on large concrete specimens. Work is in progress for the further improvement of its performance.

\section{References}

1. P.H. Bischoff, S.H. Perry: Mater Struct 24 (1991)

2. E. Cadoni, G. Solomos, C. Albertini: Mag Concrete Res 60 (2008)

3. G.T. Gray III: ASM handbook Mechanical testing and evaluation, 8 (2000)

4. W.W. Chen, B.Song: Split Hopkinson (Kolsky) Bar: Design, Testing and Applications, Springer-Mechanical Engineering Series (2010)

5. H. Zhao, G. Gary: J. Mech. Phys. Solids 45 (1997)

6. A. Tyas, A.J. Watson: Int. J. Impact Eng. 25 (2001)

7. G. Gary, K. Safa:, Int. J. Impact Eng. 37 (2010) 